\title{
Assessment of Yield Losses due to Shoot and Fruit Borer, Earias vittella (Fab.) in Okra
}

\author{
Anurag Potai*, Gajendra Chandrakar and Sneha Tiwari
}

Department of Entomology, College of Agriculture, Indira Gandhi Krishi Vishwavidyalaya, Raipur, Chhattisgarh, India-492012

*Corresponding author

\section{Keywords}

Shoot and Fruit

Borer, BAS 45001

I $300 \mathrm{SC}$,

Chlorantraniliprole $18.5 \% \mathrm{SC}$,

Cypermethrin $10 \%$ EC, Okra

Article Info

Accepted:

12 May 2019

Available Online:

10 June 2019

\section{A B S T R A C T}

Experimentation was done to observe the efficacy of some insecticides against shoot and fruit borer, Earias vittella (Fabricius) of okra during kharif season 2016 at the Horticulture farm, Department of Horticulture, College of Agriculture, Indira Gandhi Krishi Vishwavidyalaya (IGKV) Raipur, Chhattisgarh. The insecticidal treatments BAS 45001 I 300 SC @18.5g.a.i./ha was found to be most effective as it recorded minimum fruit damage (14.29\%) followed by Chlorantraniliprole 18.5\% SC@ 25g.a.i./ha (15.68\%). BAS 45001 I 300 SC @ 12.5g.a.i./ha (16.33\%) and BAS 45001 I 300 SC @6.5g.a.i./ha $(18.74 \%)$ was found statistically on par with each other, followed by Cypermethrin $10 \%$ EC @ 70g.a.i./ha (21.14) Maximum healthy fruit yield recorded from the treatment BAS 45001 I300 SC @ 18.5g.a.i/ha (155.99 q/ha), followed by Chlorantraniliprole18.5\% SC @25g.a.i/ha (155.10q/ha), Cypermethrin 10\% EC@70g.a.i./ha (154.99q/ha).

\section{Introduction}

Amongst the various vegetable grown Okra Abelmoschus esculentus L. (Moench) belongs to family Malvaceae, is an economically important vegetable crop grown in tropical and sub-tropical parts of the world. This crop is suitable for cultivation as a kitchen garden crop as well as on large high-tech commercial farms. It is grown commercially in India, Turkey, Iran, Western Africa, Yugoslavia, Bangladesh, Afghanistan, Pakistan, Burma, Japan, Malaysia, Brazil, Ghana, Ethiopia and the Southern United States. Vegetables constitute an important food item, supplying vitamins, carbohydrates and minerals needed for a balanced diet (Randhawa, 1974; Masood Khan et al., 2001).

There are several constraints in the cultivation of okra. Many of the pests occurring on cotton are also found on okra crop. As high as, 72 species of insects have been recorded on okra (Srinivas Rao and Rajendran, 2002), of which, the sucking pests comprising of leafhopper, Amrasca biguttula biguttula (Ishida), whitefly, Bemisia tabaci (Gennadius) and mite, Tetranychus urticae 
(Boisduval) cause significant damage to the crop. Leafhopper, a polyphagous, pest has been a serious pest on okra causing heavy loss during these years. High population of leafhopper significantly sucks cell sap usually from ventral surface of the leaves and inject toxic saliva into plant tissues, turning the leaves to yellowish and curl upward (Singh et $a l ., 2008)$. Whitefly (B. tabaci) nymphs and adults remove significant amount of cell sap from the leaves to reduce the plant vigour. They are responsible for transmitting yellow vein mosaic virus also. Red spider mites scratch the leaf tissues and lap the oozing out sap. Heavy webbing caused by the mite make it difficult to control.

The crop sustains different insect fauna which serve to attract numerous predators (Bilal and Satti, 2012). To overcome these problems, identification of new molecules is the need of the hour so as to fit them in IPM practices. Natural enemies co-exist with the pests in a crop ecosystem. Heavy use of insecticides with high toxicity can control the pest effectively but it may have adverse effect on natural enemies' population. Hence, the effects of different insecticides on population of natural enemies were studied and results of present findings were discussed here under.

\section{Materials and Methods}

The experimental site was conducted at Horticulture farm, Department of Horticulture, College of Agriculture, Indira Gandhi Krishi Vishwavidyalaya (IGKV) Raipur, Chhattisgarh. During 2016-17 Kharif season to study the effect of different insecticides against shoot and fruit borer. Super green variety of okra seeds were sown in the field with a spacing of $60 \times 15 \mathrm{~cm}$ between rows and plants, respectively with a plot size of $5 \mathrm{mx} 4 \mathrm{~m} .=20 \mathrm{~m} 2$ in a Randomized Block Design (RBD) consisting of six treatments and replicated four times. The crops were raised following all recommended agronomic practices. Observations was recorded on 10 randomly selected plants/ plot before fruiting and thereafter total number of damaged fruits will be counted at each picking.

\section{Statistical analysis of data}

Data on per cent fruit and shoot borer infestation were transformed to Arcsine transformation before statistical analysis

Percent of fruit damage (q/ha)were worked out with the help of following formula:

Number of damaged fruits

Per cent fruit damage $=-100$

Total number of fruits (healthy + Damage)

Weight of damaged fruits

Per cent yield loss $=-\times 100$

Total yield of fruits (healthy + Damage)

\section{Results and Discussion}

\section{Effect of different doses of newer insecticides against shoot and fruit borer of insect pest on okra}

During the okra crop, novel approaches of insect management by using new molecules of insecticides may play important role to manage shoot and fruit borer and safer to non target pest and natural enemies. The computed data of mean per cent of fruit damage indicated that, the plot treated twice with BAS 45001 I 300 SC 18.5g.a.i./h were significantly effective with minimum fruit damage of 14.29 percent, followed by Chlorantranilip role 18.5\% SC @25g.a.i./ha (15.68\%), BAS $450 \quad 01 \quad$ I $300 \quad$ SC @ 12.5g.a.i./h (16.33\%), BAS 45001 I 300 SC@6.5g.a.i./h (18.74\%), Cypermethrin 10\% EC @70g.a.i./ha (21.14\%), respectively, higher per cent fruit infestation were recorded from untreated control $\left(\mathrm{T}_{6}\right)(25.84 \%)$ (Table 1 and 2). 
Table.1 Bioefficacy of BAS 45001 I 300 SC against shoot and fruit borer of okra

\begin{tabular}{|c|c|c|c|c|c|c|c|c|c|c|c|c|}
\hline \multirow[t]{3}{*}{ Insecticide } & \multirow[t]{3}{*}{ Dose } & \multicolumn{10}{|c|}{ Percent shoot and fruit borer infestation in different fruit picking } & \multirow{3}{*}{$\begin{array}{l}\text { Mean \% } \\
\text { fruit } \\
\text { Damage }\end{array}$} \\
\hline & & $\begin{array}{l}26 / 08 / 201 \\
6\end{array}$ & $30 / 08$ & $6 / 09$ & 09/09 & $14 / 09$ & $16 / 09$ & 19/09 & $26 / 09$ & $03 / 10$ & 07/10 & \\
\hline & & $1^{\text {st }}$ Peaking & $2^{\text {nd }}$ & $3^{\text {rd }}$ & $4^{\text {th }}$ & $5^{\text {th }}$ & $6^{\text {th }}$ & $7^{\text {th }}$ & $8^{\text {th }}$ & $9^{\text {th }}$ & $10^{\text {th }}$ & \\
\hline $\begin{array}{l}\text { BAS } 45001 \text { I } 300 \\
\text { SC }\end{array}$ & $\begin{array}{l}6.5 \mathrm{~g} \\
\text { a.i./ha }\end{array}$ & $\begin{array}{c}8.03 \\
(14.89)\end{array}$ & $\begin{array}{c}10.59 \\
(18.39)\end{array}$ & $\begin{array}{c}13.95 \\
(21.60)\end{array}$ & $\begin{array}{c}15.08 \\
(22.64)\end{array}$ & $\begin{array}{c}18.58 \\
(25.49)\end{array}$ & $\begin{array}{c}21.33 \\
(27.48)\end{array}$ & $\begin{array}{c}24.08 \\
(29.36)\end{array}$ & $\begin{array}{c}22.83 \\
(28.53)\end{array}$ & $\begin{array}{c}25.08 \\
(30.01)\end{array}$ & $\begin{array}{c}27.83 \\
(31.82)\end{array}$ & 18.74 \\
\hline $\begin{array}{l}\text { BAS } 45001 \text { I } 300 \\
\text { SC }\end{array}$ & $\begin{array}{l}12.5 \mathrm{~g} \\
\text { a.i./ha }\end{array}$ & $\begin{array}{c}4.40 \\
(12.08)\end{array}$ & $\begin{array}{c}6.05 \\
(14.19)\end{array}$ & $\begin{array}{c}10.05 \\
(18.47)\end{array}$ & $\begin{array}{c}12.16 \\
(20.33)\end{array}$ & $\begin{array}{c}16.41 \\
(23.87)\end{array}$ & $\begin{array}{c}19.66 \\
(26.21)\end{array}$ & $\begin{array}{c}23.16 \\
(28.75)\end{array}$ & $\begin{array}{c}21.91 \\
(27.88)\end{array}$ & $\begin{array}{c}24.16 \\
(29.42)\end{array}$ & $\begin{array}{c}25.30 \\
(30.18)\end{array}$ & 16.33 \\
\hline $\begin{array}{l}\text { BAS } 45001 \text { I } 300 \\
\text { SC }\end{array}$ & $\begin{array}{l}18.5 \mathrm{~g} \\
\text { a.i./ha }\end{array}$ & $\begin{array}{c}3.76 \\
(10.64)\end{array}$ & $\begin{array}{c}6.13 \\
(14.07)\end{array}$ & $\begin{array}{c}8.13 \\
(16.28)\end{array}$ & $\begin{array}{c}10.59 \\
(18.92)\end{array}$ & $\begin{array}{c}13.09 \\
(21.15)\end{array}$ & $\begin{array}{c}16.84 \\
(24.14)\end{array}$ & $\begin{array}{c}19.59 \\
(26.25)\end{array}$ & $\begin{array}{c}19.09 \\
(25.89)\end{array}$ & $\begin{array}{c}21.84 \\
(27.83)\end{array}$ & $\begin{array}{c}23.84 \\
(29.20)\end{array}$ & 14.29 \\
\hline $\begin{array}{l}\text { Chlorantraniliprole } \\
18.5 \% \text { SC }\end{array}$ & $\begin{array}{l}25 \mathrm{~g} \\
\text { a.i./ha }\end{array}$ & $\begin{array}{c}4.49 \\
(12.11)\end{array}$ & $\begin{array}{c}7.19 \\
(15.54)\end{array}$ & $\begin{array}{c}9.44 \\
(17.84)\end{array}$ & $\begin{array}{c}11.55 \\
(19.69)\end{array}$ & $\begin{array}{c}15.30 \\
(23.01)\end{array}$ & $\begin{array}{c}18.55 \\
(25.42)\end{array}$ & $\begin{array}{c}21.05 \\
(27.28)\end{array}$ & $\begin{array}{c}19.80 \\
(26.40)\end{array}$ & $\begin{array}{c}23.05 \\
(28.66)\end{array}$ & $\begin{array}{c}26.40 \\
(30.89)\end{array}$ & 15.68 \\
\hline $\begin{array}{l}\text { Cypermethrin } 10 \% \\
\text { EC }\end{array}$ & $\begin{array}{l}70 \mathrm{~g} \\
\text { a.i./ha }\end{array}$ & $\begin{array}{c}9.01 \\
(17.32)\end{array}$ & $\begin{array}{c}11.58 \\
(19.88)\end{array}$ & $\begin{array}{c}14.14 \\
(22.07)\end{array}$ & $\begin{array}{c}17.56 \\
(24.73)\end{array}$ & $\begin{array}{c}21.06 \\
(27.29)\end{array}$ & $\begin{array}{c}24.31 \\
(29.52)\end{array}$ & $\begin{array}{c}27.31 \\
(31.47)\end{array}$ & $\begin{array}{c}26.06 \\
(30.66)\end{array}$ & $\begin{array}{c}29.06 \\
(32.60)\end{array}$ & $\begin{array}{c}31.31 \\
(34.00)\end{array}$ & 21.14 \\
\hline Untreated control & - & $\begin{array}{c}14.14 \\
(22.07)\end{array}$ & $\begin{array}{c}14.73 \\
(22.56)\end{array}$ & $\begin{array}{c}17.12 \\
(24.42)\end{array}$ & $\begin{array}{c}20.02 \\
(26.56)\end{array}$ & $\begin{array}{c}24.77 \\
(29.83)\end{array}$ & $\begin{array}{c}27.02 \\
(31.30)\end{array}$ & $\begin{array}{c}31.02 \\
(33.81)\end{array}$ & $\begin{array}{c}33.77 \\
(35.51)\end{array}$ & $\begin{array}{c}36.27 \\
(37.01)\end{array}$ & $\begin{array}{c}39.52 \\
(38.93)\end{array}$ & 25.84 \\
\hline $\begin{array}{l}\text { Sem } \pm \\
\text { CD at 5\% }\end{array}$ & & $\begin{array}{l}1.73 \\
5.27\end{array}$ & $\begin{array}{l}1.29 \\
3.94\end{array}$ & $\begin{array}{l}1.14 \\
3.47\end{array}$ & $\begin{array}{l}1.12 \\
3.42\end{array}$ & $\begin{array}{l}0.64 \\
1.94\end{array}$ & $\begin{array}{l}0.79 \\
2.42\end{array}$ & $\begin{array}{l}0.61 \\
1.85\end{array}$ & $\begin{array}{l}0.57 \\
1.75\end{array}$ & $\begin{array}{l}0.60 \\
1.83\end{array}$ & $\begin{array}{l}0.49 \\
1.49\end{array}$ & $\begin{array}{l}0.90 \\
2.74\end{array}$ \\
\hline
\end{tabular}

* Figure is parenthesis in Arcsine transformed values 
Table.2 Per cent reduction in yield due to infestation of E. vittella in different treatments of okra during Kharif 2016-17

\begin{tabular}{|c|c|c|c|c|c|c|}
\hline \multirow[t]{2}{*}{ S. No. } & \multirow[t]{2}{*}{ Name of treatment } & \multirow[t]{2}{*}{ Dose } & \multicolumn{3}{|c|}{$\begin{array}{c}\text { Fruit yield } \\
\text { (q/ha) }\end{array}$} & \multirow{2}{*}{$\begin{array}{l}\text { Reduction } \\
\text { in yield } \\
(\%)\end{array}$} \\
\hline & & & Healthy & Damage & Total & \\
\hline $\mathbf{T}_{1}$ & BAS 45001 I 300 SC & $\begin{array}{l}6.5 \mathrm{~g} \\
\text { a.i./ha }\end{array}$ & 153.21 & 4.42 & 157.63 & 3.32 \\
\hline $\mathbf{T}_{2}$ & BAS 45001 I 300 SC & $\begin{array}{l}12.5 \mathrm{~g} \\
\text { a.i./ha }\end{array}$ & 154.11 & 2.39 & 156.50 & 4.01 \\
\hline $\mathbf{T}_{\mathbf{3}}$ & BAS 45001 I 300 SC & $\begin{array}{l}18.5 \mathrm{~g} \\
\text { a.i./ha }\end{array}$ & 155.99 & 2.12 & 158.11 & 3.02 \\
\hline $\mathbf{T}_{4}$ & $\begin{array}{c}\text { Chlorantraniliprole } \\
18.5 \% \text { SC }\end{array}$ & $\begin{array}{l}25 \mathrm{~g} \\
\text { a.i./ha }\end{array}$ & 155.10 & 2.79 & 157.89 & 3.16 \\
\hline $\mathbf{T}_{5}$ & $\begin{array}{c}\text { Cypermethrin } 10 \% \\
\text { EC }\end{array}$ & $\begin{array}{l}70 \mathrm{~g} \\
\text { a.i./ha }\end{array}$ & 154.99 & 3.65 & 158.64 & 2.70 \\
\hline $\mathbf{T}_{6}$ & Untreated control & - & 153.00 & 10.05 & 163.05 & \\
\hline
\end{tabular}

Patra et al., (2009) reported that, Emamectin benzoate 5SG@15g.a.i/ha recorded lowest (4.7\%) shoot infestation which was at par with Spinosad $2.5 \quad$ SC@50g.a.i./ha (4.9\%).This was followed by Indoxacarb 14.5 SC@50g.a.i./ha (5.2\%) and Methoxyfenzide 24\%SC 200g.a.i./ha (7.1\%). The highest shoot infestation was recorded in untreated check (21.2\%). Sharma and Kaushik (2010) evaluated Spinosad 45 SC along with six chemical insecticides and reported that, Spinosad $45 \mathrm{SC}(162.5 \mathrm{ml} / \mathrm{ha})$ was most effective against shoot and fruit borer compare to other six chemical insecticides.

Per cent reduction in yield of okra due to infestation of shoot and fruit borer $E$. vittella in different treatments

On the basis of fruit yield of healthy fruits of okra ranged between 153.00 and 155.99 q/ha. Maximum healthy fruit yield recorded from the treatment BAS $450 \quad 01 \quad$ I300 SC @ 18.5g.a.i/ha (155.99 q/ha), followed by Chlorantraniliprole18.5\% SC @25g.a.i/ha (155.10q/ha), Cypermethrin $\quad 10 \%$ EC@70g.a.i./ha (154.99q/ha), BAS 45001
I300 SC @12.5g.a.i/ha (154.11 q/ha), BAS 45001 I300 SC @6.5g.a.i./ha (153.21 q/ha). The lowest healthy fruit total yield recorded from untreated control $\left(\mathrm{T}_{6}\right)(153.00 \mathrm{q} / \mathrm{ha})$.

Kuttalam et al., (2008) were tested Emamectin benzoate 5EC in four doses i.e. 9, 11, 13 and 15 g.a.i./ha against Earias vittella (Fab) at Coimbatore.

\section{References}

Masood Khan, M.A., Jagadishwar Reddy, D. and Venkateshwar Rao, S. 2001, Bioefficacy of selected insecticides against pest complex in okra. Pestology, 26 (6): 18-23.

Randhawa, G.S., 1974, Horticulture; Importance of pest control. Pesticides Annual, pp. 85-87.

Singh S, Choudhary DP, Sharma HC, Mahla RS, Mathur YS. Ahuja DB.2008. Effect of insecticidal modules against jassid and shoot and fruit borer in okra. Indian J. Entomol. 70(3): 197199.

Bilal, N.A. and Satti, A.A. 2012. The major 
predators and their seasonal abundance in okra fields at Elgorair scheme, Northern Sudan. The experiment, 4(4): 271-276.

Srinivasa, Rao, N. and Rajendra, R., 2002, Joint action potential of neem with other plant extracts against the leaf hoppers, Amrasca devastanse (Distant) on okra. Pest Management and Economic Zoology, 10:131-136.

Kuttalam, s., Boomathi, N., Kumar, B.V. and Rajathi, S., 2008, Field efficacy of Emamectin benzoate 5EC against okra fruit borer, Earias vittella (Fab.). Pestology, 32: 32-36.

Patra, S., Chatterjee, M.L. and Samanta, A., 2009, Field efficacy of insecticides for management of Earias vittella in okra. Ann.Pl.Protec.Sci., 17(2):459-526.

Sharma, S.S. and Kaushik, H.D., 2010, Effect of Spinosad (a bioinsecticide) and other insecticides against pest complex and natural enemies on eggplant (Solanum melongena L.). Journal of Entomological Research. 34(1): 39-44.

\section{How to cite this article:}

Anurag Potai, Gajendra Chandrakar and Sneha Tiwari. 2019. Assessment of Yield Losses due to Shoot and Fruit Borer, Earias vittella (Fab.) in Okra. Int.J.Curr.Microbiol.App.Sci. 8(06): 1303-1307. doi: https://doi.org/10.20546/ijcmas.2019.806.157 